\title{
\begin{tabular}{l|l} 
Mibraries & DSpace@MIT
\end{tabular}
}

\author{
MIT Open Access Articles
}

Ground calibration of the Silicon Drift Detectors for NICER

The MIT Faculty has made this article openly available. Please share how this access benefits you. Your story matters.

Citation: LaMarr, B. et al. "Ground Calibration of the Silicon Drift Detectors for NICER." Space Telescopes and Instrumentation 2016: Ultraviolet to Gamma Ray, June - July 2016, Edinburgh, United Kingdom, SPIE, July 2016 @ 2016 SPIE

As Published: http://dx.doi.org/10.1117/12.2232784

Publisher: SPIE

Persistent URL: http://hdl.handle.net/1721.1/115079

Version: Final published version: final published article, as it appeared in a journal, conference proceedings, or other formally published context

Terms of Use: Article is made available in accordance with the publisher's policy and may be subject to US copyright law. Please refer to the publisher's site for terms of use. 


\section{Ground calibration of the Silicon Drift Detectors for NICER}

Beverly LaMarr, Gregory Prigozhin, Ronald Remillard, Andrew Malonis, Keith C. Gendreau, et al.

Beverly LaMarr, Gregory Prigozhin, Ronald Remillard, Andrew Malonis, Keith C. Gendreau, Zaven Arzoumanian, Craig B. Markwardt, Wayne H. Baumgartner, "Ground calibration of the Silicon Drift Detectors for NICER," Proc. SPIE 9905, Space Telescopes and Instrumentation 2016: Ultraviolet to Gamma Ray, 99054W (19 July 2016); doi: 10.1117/12.2232784

Event: SPIE Astronomical Telescopes + Instrumentation, 2016, Edinburgh, United Kingdom 


\title{
Ground Calibration of the Silicon Drift Detectors for NICER
}

\author{
Beverly LaMarr ${ }^{\mathrm{a}}$, Gregory Prigozhin ${ }^{\mathrm{a}}$, Ronald Remillard ${ }^{\mathrm{a}}$, Andrew Malonis ${ }^{\mathrm{a}}$, Keith C. \\ Gendreau $^{\mathrm{b}}$, Zaven Arzoumanian ${ }^{\mathrm{b}}$, Craig B. Markwardt ${ }^{\mathrm{b}}$, and Wayne H. Baumgartner ${ }^{\mathrm{b}}$ \\ ${ }^{a}$ MIT Kavli Institute for Astrophysics, Cambridge, MA, USA \\ bNASA Goddard Space Flight Center, Greenbelt, MD, USA
}

\begin{abstract}
The Neutron star Interior Composition ExploreR (NICER) is set to be deployed on the International Space Station (ISS) in early 2017. It will use an array of 56 Silicon Drift Detectors (SDDs) to detect soft X-rays (0.2 $12 \mathrm{keV}$ ) with 100 nanosecond timing resolution. Here we describe the effort to calibrate the detectors in the lab primarily using a Modulated X-ray Source (MXS).

The MXS that was customized for NICER provides more than a dozen emission lines spread over the instrument bandwidth, providing calibration measurements for detector gain and spectral resolution. In addition, the fluorescence source in the MXS was pulsed at high frequency to enable measurement of the delay due to charge collection in the silicon and signal processing in the detector electronics. A second chamber, designed to illuminate detectors with either ${ }^{55} \mathrm{Fe}$, an optical LED, or neither, provided additional calibration of detector response, optical blocking, and effectiveness of background rejection techniques. The overall ground calibration achieved total operating time that was generally in the range of 500-1500 hours for each of the 56 detectors.
\end{abstract}

Keywords: Silicon Drift Detectors, X-rays, timing spectroscopy, calibration

\section{INTRODUCTION}

The Instrument Detector Subsystem of the Xray Timing Instrument (XTI $)^{1}$ on NICER ${ }^{2}$ includes 56 SDDs. $^{3}$ All of these, and spares, were characterized at MIT in 2015 using an MXS. ${ }^{4}$ The source included lines from $277 \mathrm{eV}$ to $8.9 \mathrm{keV}$ pulsed at $600 \mathrm{kHz}$.

The fluorescence pulses were driven by a function generator, commanded to deliver square waves with a duration of $20 \mathrm{~ns}$. The illumination time per pulse is faster than the clock ticks (approximately $38 \mathrm{~ns}$ ) in the Measurement \& Power Unit (MPU). The MPU clock was synchronized to the MXS pulse generator at each second boundary, so that the delay between measured event times and the actual x-ray pulse time can be measured.

Each of the seven identical MPUs in the XTI services eight SDDs, and the signal processing designs for the eight channels are described elsewhere ${ }^{1}$ in these proceedings. Each channel is further equipped with two signal processing chains, distinguished by "fast" and "slow" shaping times, which are optimized to record events times and pulse amplitudes (for spectra), respectively.

The SDD signal line is replicated in the MPU and then fed to the fast and slow measuring chains, in parallel. Signal variations separately trigger fast and slow chains, and events are recorded when one or both chains produces a trigger. The telemetered values for each event include the MPU and channel IDs, trigger flags from each chain, event time (preferably from the fast chain, when triggered), integrated pulse amplitudes from each chain, the deadtime for that event, and additional flags that warn of high amplitude (i.e, non-X-ray) events that trip "overflow" and "underflow" thresholds included in the hardware. Finally, the MPU can be commanded to sample and process the signal line at any arbitrary time. Such "forced trigger" events are an effective way to monitor the event amplitudes values associated with $0 \mathrm{keV}$.

Space Telescopes and Instrumentation 2016: Ultraviolet to Gamma Ray, edited by

Jan-Willem A. den Herder, Tadayuki Takahashi, Marshall Bautz, Proc. of SPIE Vol. 9905, 99054W

(C) 2016 SPIE · CCC code: 0277-786X/16/\$18 · doi: 10.1117/12.2232784

Proc. of SPIE Vol. 990599054 W-1 

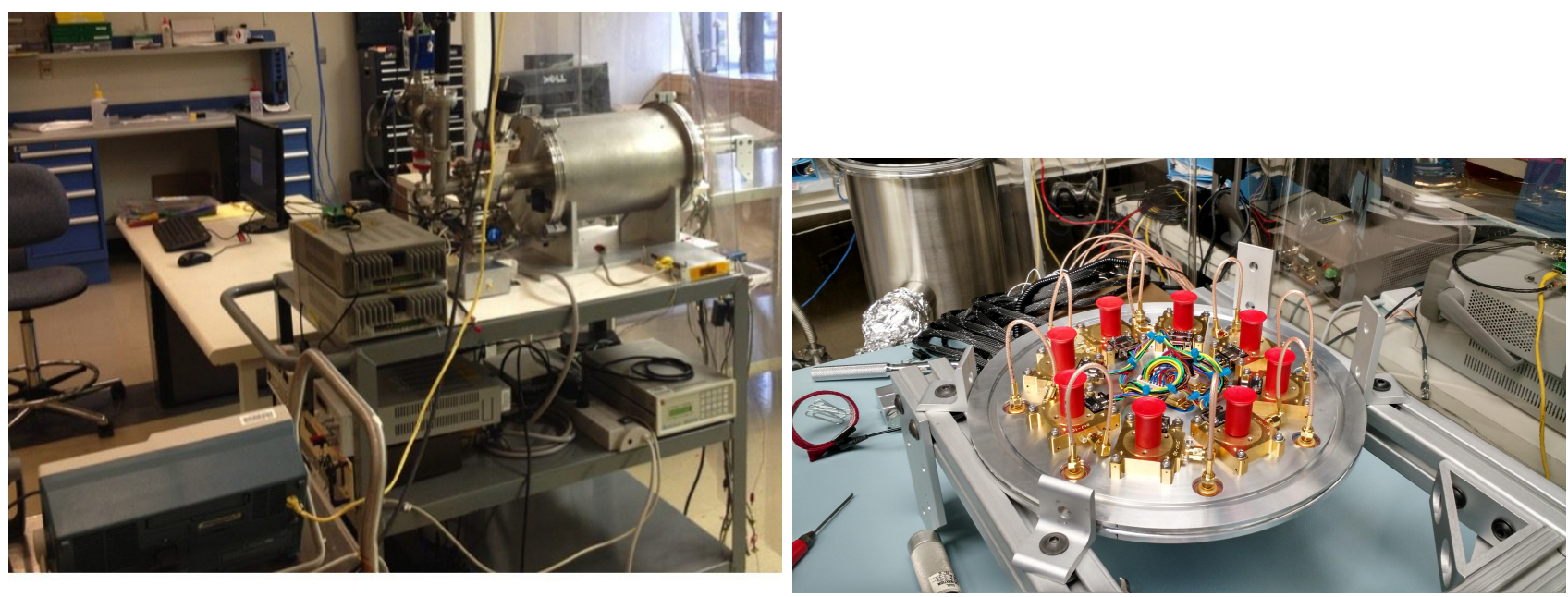

Figure 1. MXS setup: The left panel shows the vacuum tank connected to the engineering electronics and the MXS with its associated power supplies and pulse generator. The MXS is on the left in the figure and the detectors are mounted to the right. The right panel shows the plate with flight candidate FPMS mounted.

\section{SETUP}

The detectors were tested eight at a time in a vacuum chamber attached to the MXS. An engineering, flight like, MPU was used to readout the detectors using the same data formats as planned for flight.

The MXS chamber and the chamber base plate are shown in Figure 1. During MXS Calibration runs, we cycled through lots of six "new" detectors plus two references detectors that were slotted in a fixed and variable locations on the base pate, respectively. The reference detector with variable position allowed us to correct for inevitable beam differences for each emission line on the multi-element target, while the fixed reference detector primarily monitored evolution in the brightness of the lines.

As noted above, the ground Calibration utilized a second Chamber, designed to collect spectra from an ${ }^{55} \mathrm{Fe}$ source, an optical LED, or simply the ambient background. The second chamber allowed deep calibration of the detector response to ${ }^{55} \mathrm{Fe}$, measurement of the optical blocking characteristics for each detector window, and tests of the effectiveness of background rejection techniques. The second chamber was run overnight or on weekends (in ${ }^{55} \mathrm{Fe}$ or background modes), since the detectors were not in vacuum, and there was no need to monitor against variations in the illumination source. The combined set of both chambers allowed us to reach total operating times of generally 500-1500 hours for each of the 56 detectors. Calibration runs spanned 2014 November through 2015 August at MIT.

\section{SPECTRAL CHARACTERIZATION}

\subsection{Energy Scale}

The MXS provides us with pulsed x-rays at multiple energies, from $0.28 \mathrm{keV}$ to $8.9 \mathrm{keV}$, simultaneously.

A sample MXS spectrum, in events versus slow-chain amplitude (ADU) is given in the left panel of Figure 2. The gain curve, determined from the relation between the line centers (ADU) and line energies (keV) is illustrated in the right panel of Figure 2.

Further author information: (Send correspondence to BL)

BL: E-mail: fergason@space.mit.edu 

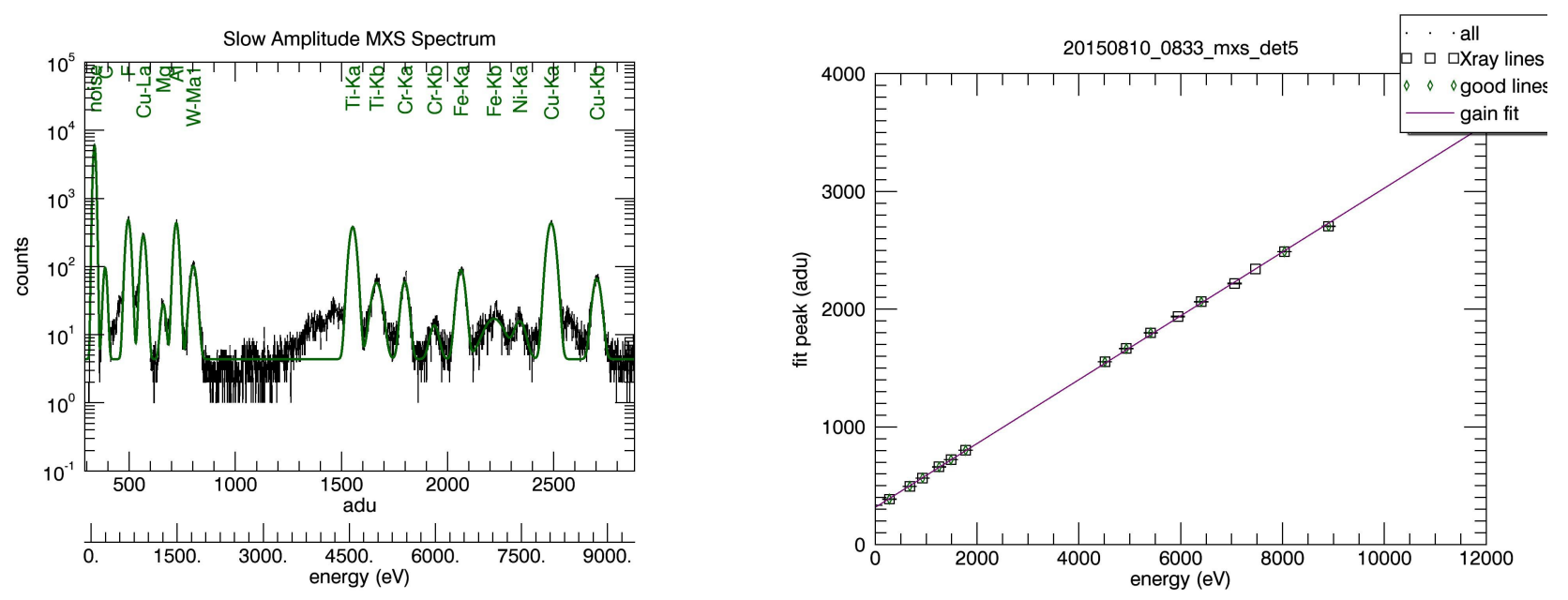

Figure 2. Slow channel spectrum

\subsection{Energy Resolution}

The same fits that provide the gain values in section 3.1 also provide line widths. The right panel of figure 3 provides the full width at half maximum (FWHM) for all of the flight detectors as measured in the calibration setup. The solid lines are a fit to the points that resulted in the noise values in the histogram on the left.

The results in Figure 3 show that the detectors all meet the energy resolution requirement for NICER: FWHM less than $120 \mathrm{eV}$ from 0.2 to $1.0 \mathrm{keV}$ and less than $200 \mathrm{eV}$ from 1.0 to $12.0 \mathrm{keV}$.
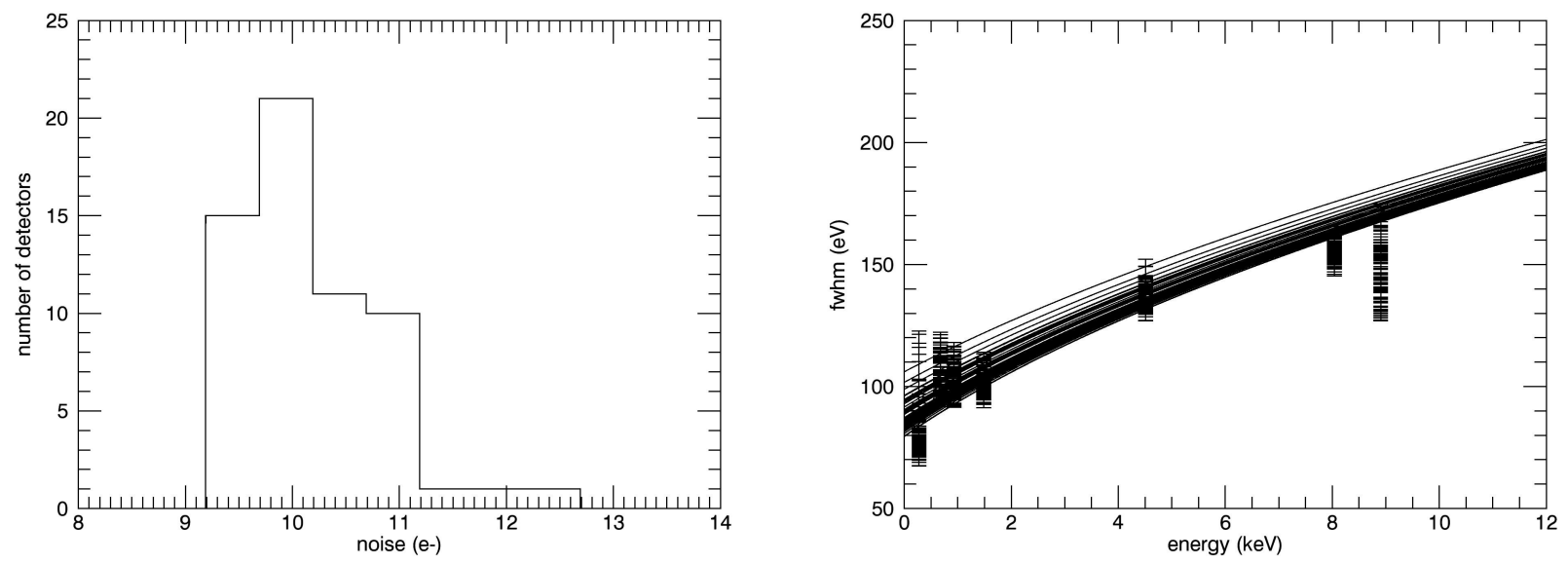

Figure 3. Slow channel spectral resolution

\subsection{Forced Triggers}

As noted in the introduction, when configured to do so, the electronics will periodically force a trigger. The zero level and noise of the amplitude values can be measured by treating this as an event. Figure 4 shows histograms of the width of the force trigger peaks for both the slow and fast channels for all flight detectors. The forced trigger values are easily separated from other events because there is a flag set for these events. 

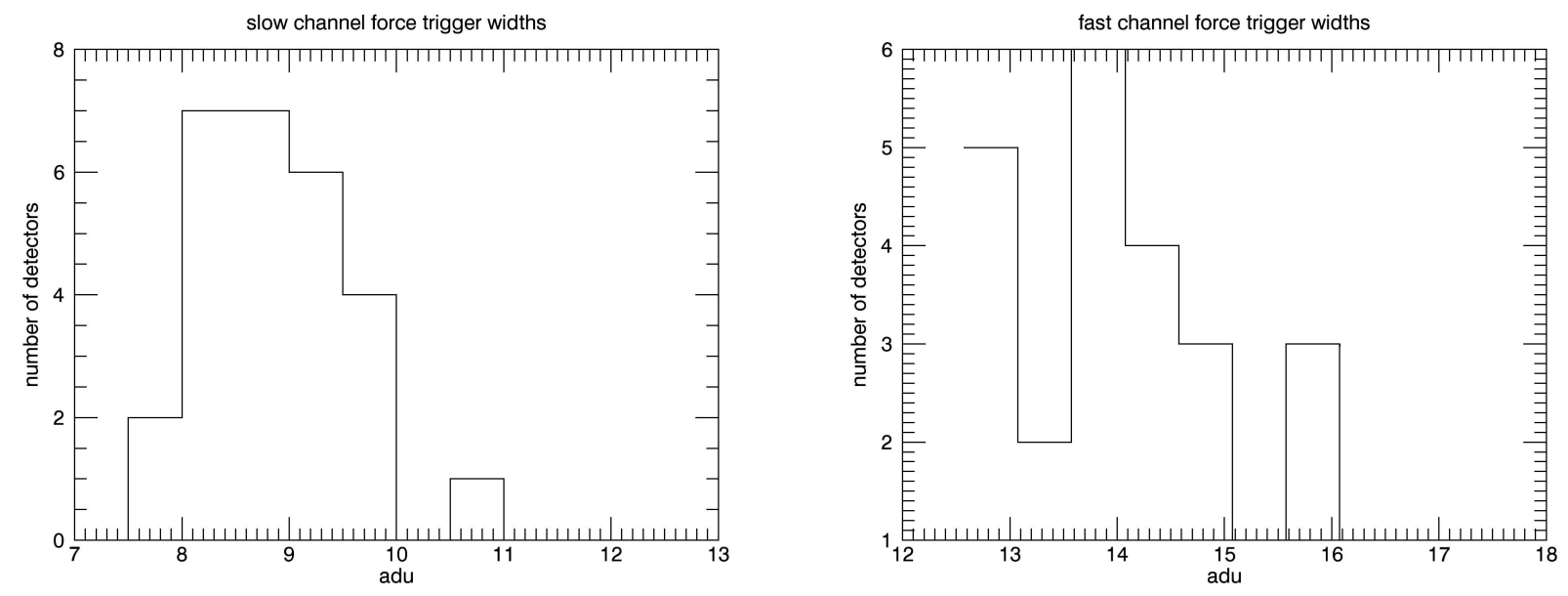

Figure 4. Forced Trigger Widths: The left panel is the sigma of a gaussian a fit to the slow amplitude of force trigger events. The right panel is for a fit to the fast amplitude values of the same events.

\section{TIMING CHARACTERIZATION}

\subsection{Event Timing}

Event times are recorded as an MPU tick number, and the accuracy of the MPU clock is maintained with an external GPS interface. At each GPS second, the MPU receives a tone and then captures the MPU tick number at the time of the tone. The MPU then receives the GPS time at the last tone, and the log of tick number versus GPS seconds is later used to correct the MPU time history for drifts, variations due to MPU temperature, and other possible deviations. Uncertainties in all sources must remain within the NICER requirement to time-tag events in the Solar Barycenter to an accuracy less than $100 \mathrm{~ns}$.

In the MXS setup, the same pulse that is used to lock the time is also used to start $600 \mathrm{kHz}$ pulses of the $\mathrm{X}$-rays. Knowing this, we can calculate the time since the x-ray pulse for each event. The left panel of figure 5 is the image of a two dimensional histogram of time since the x-ray pulse and the slow event amplitude for one detector. The delay time averages generally represent the detector time offset for the fast chain, which takes precedence in time tagging (despite the fact that the results are plotted versus slow chain event amplitude). The average offsets do not vary as a function of line energy, except for the very first line at $277 \mathrm{eV}$ (near edge of the left panel plot). Here, the noisier fast chain is not triggering for these events (fast trigger $=0$ ), the slow chain time is alternatively used, and the jump in the time offset reflects the properties of the slow chain electronics. During calibration, the time offsets for the slow chain were mapped at all x-ray energies (not shown) simply by repeating the measurements while raising the trigger threshold for the fast chain to a very high level, effectively disabling the fast chain time tags.

The right panel of figure 5 shows the width of a histogram fit to those times for each of the main spectral lines. Each color and symbol is a different channel during a different data set. Set to set variations are clearly more important than the energy of the incident x-rays. The width of the time fits is also influenced by the 20 nanosecond length of the pulse and the uniform illumination of the full two millimeter diameter opening of the SDD collimator. Despite the variations in the widths of the time offsets (Figure 5 right), the detector system remains within the error budget for achieving the timing accuracy for NICER, as noted above.

As described elsewhere,${ }^{1}$ the fast channel shaping time is 84 nanoseconds and the slow channel shaping time is 465 nanoseconds. Because of this the fast channel is noisier and needs to be run with a threshold that begins to reduce detection efficiency below $1 \mathrm{keV}$. Events with amplitudes below this are likely to be detected only in the slow channel with a systematically later time. 

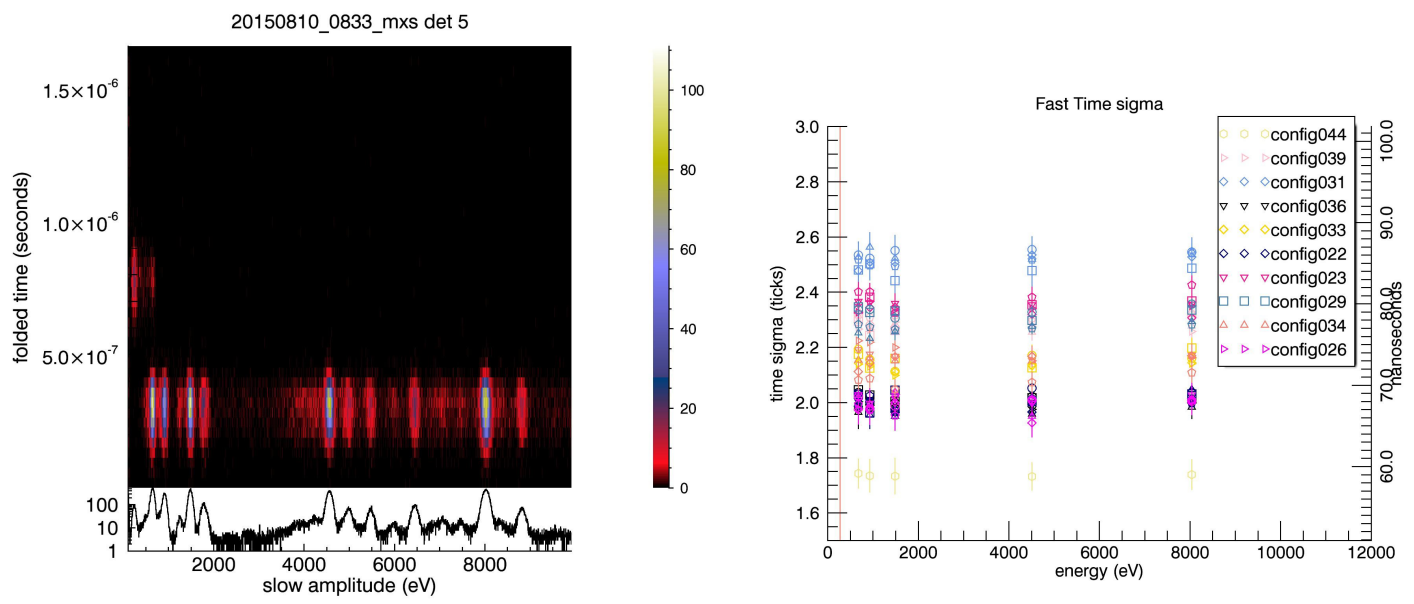

Figure 5. Event Timing: The left panel shows the times and amplitudes of detected events. The right panel shows the time resolution for each dataset at each energy.

\subsection{Deadtimes}

The deadtime for the channel associated with each event is part of the science packet. Figure 6 shows typical deadtimes for data taken with the MXS. Deadtime is traced for the different types of events, which are coded by the concatenated bits for the 5 event flags: slow trigger, fast trigger, forced trigger, overshoot, and undershoot. For example, a normal event (i.e., not forced and not surpassing undershoot or overshoot thresholds) that triggers both slow and fast chains would by type: "11000". The two main peaks seen are for events with a single channel triggered, around 15 microseconds, and for both channels triggered, around 22 microseconds. The difference between those two peaks are due to the time it takes to read the amplitude value of the second channel. Deadtimes increase when the processor is busy with other jobs such as momentarily collecting housekeeping data or generally handling events from bright sources.

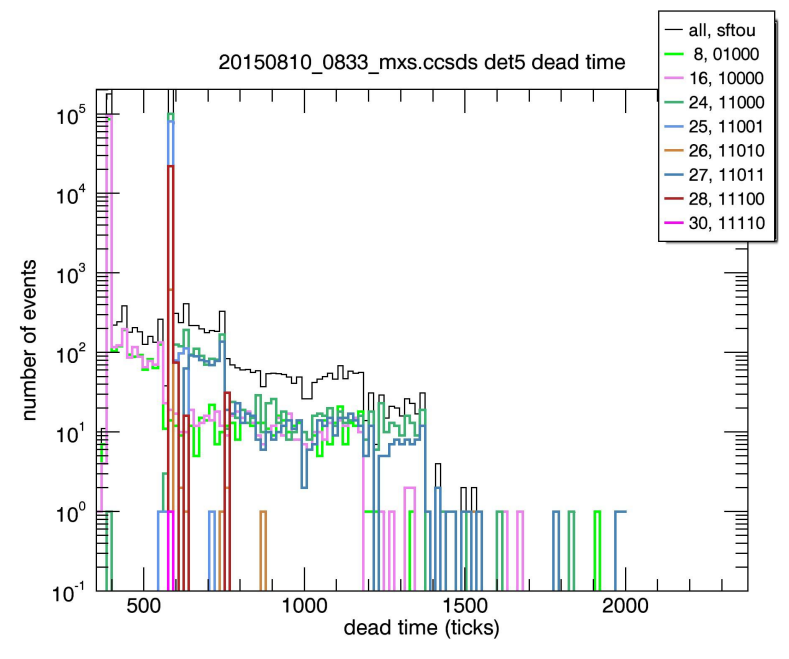

Figure 6. Deadtime for a single channel. Events are sorted by the flags that are set, slow, fast, forced, overshoot and undershoot, respectively. Each tick is approximately 38 nanoseconds. 


\section{RESETS}

The operating temperature of the SDDs is not sufficiently cold to eliminate dark current. Consequently, charge is collected in a capacitor, and it accumulates until it reaches roughly $2 \mathrm{~V}$, triggering a reset to $0 \mathrm{~V}$. When this reset occurs an event is detected which has both undershoot and overshoot flags set. These events are allowed to remain in the telemetry stream because that gives them a timestamp with the same precision as the X-ray events.

Because the dark current contributes to this effect, it is temperature dependent. The number of resets per second increases as the temperatures does.

Figure 7 shows a histogram of the average reset rates for the 56 SDDs in the XTI. At nominal operating temperature of $-55 \mathrm{C}$, the large majority of detectors have reset rates in the range $2-3 \mathrm{~Hz}$, while none exceed 10 $\mathrm{Hz}$.

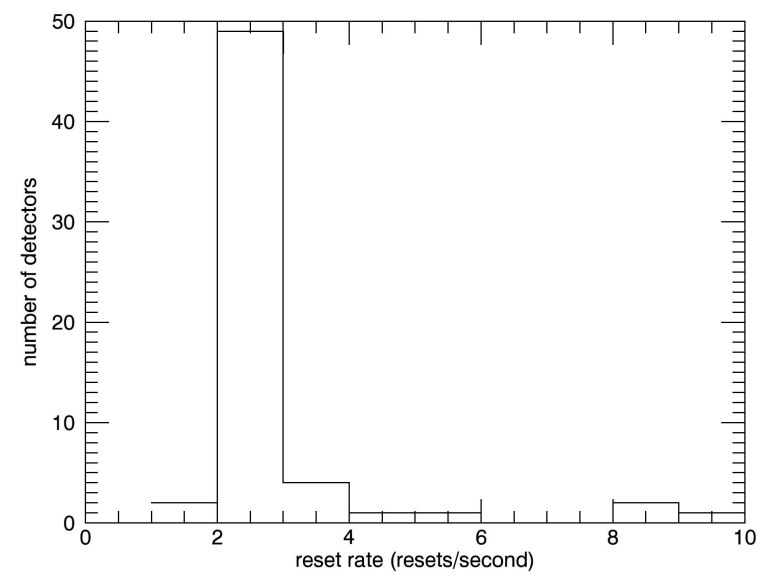

Figure 7. Reset times for flight detectors at $-55 \mathrm{C}$.

\section{RELATIVE QUANTUM EFFICIENCY}

\subsection{Reference Detectors}

At the beginning of the calibration two detectors (FPM 01-002 and FPM 01-010) were designated as reference detectors. These were on the detector plate in the chamber at all times, FPM 01-002 in a stationary location, FPM 01-010 in all other positions during the course of operations. The two detectors were later taken to the synchrotron facility BESSY-II ${ }^{5}$ for absolute QE calibration. Finally, reference detector FPM 01-002 was installed on the flight focal plane attached to MPU 7 (counting from 1) read out through channel 5 (counting from 0 ).

\subsection{Trigger Efficiency}

The trigger efficiency effect is discussed in detail elsewhere in these proceedings. ${ }^{1}$ It is an MPU effect illustrated in figure 9. Using a pulser as the input to the MPU instead of the FPM, a known number of pulses over a range of energies is fed to the chain. The three thresholds shown here represent a range that produces noise counts of less than one per second to more than a hundred counts per second when an FPM is present. The amplitude range over which the detected count rate changes is equivalent to approximately $100 \mathrm{eV}$ in all cases. Typical thresholds set during the MXS data collection are best represented by the middle curve, which begins to rise from zero counts at approximately $200 \mathrm{eV}$ and flattens by approximately $300 \mathrm{eV}$.

\section{ACKNOWLEDGMENTS}

This work was funded by NASA contract NNG14PJ13C. 

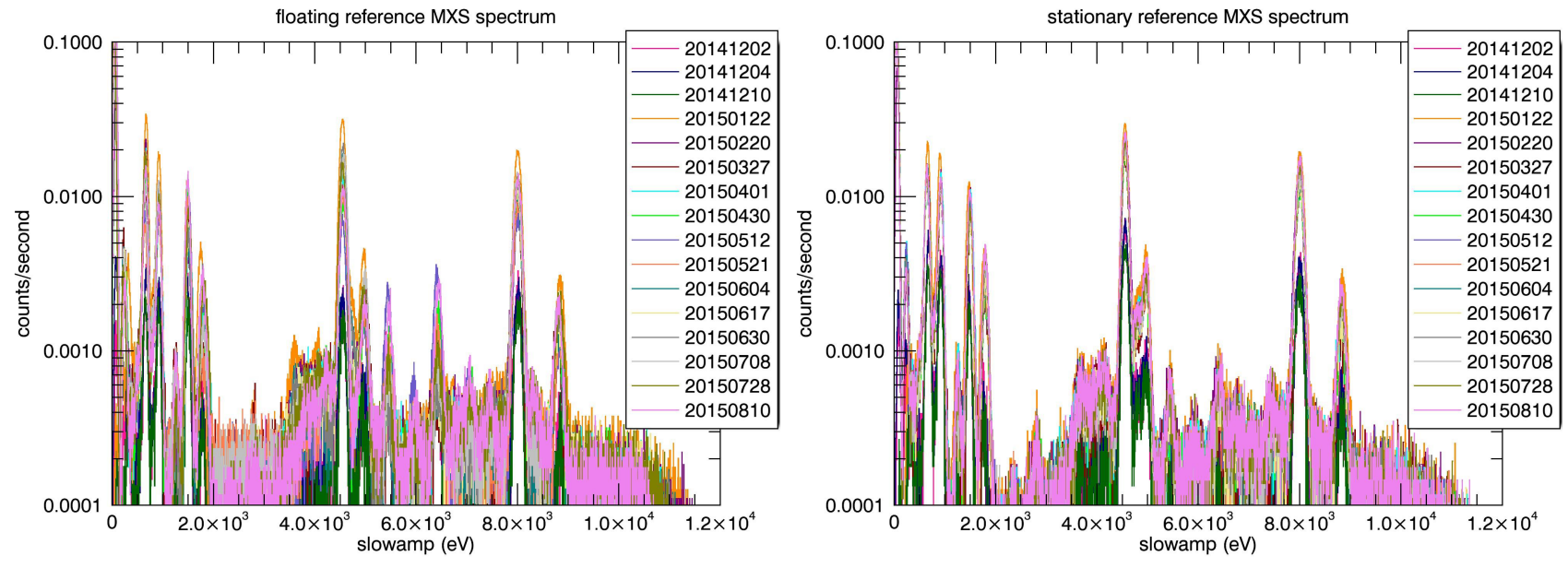

Figure 8. Reference Spectra: The left panel shows the spectra seen by the floating reference detector in each of the nominal MXS sets. The right panel shows the spectra seen by the stationary reference detector in each of the nominal MXS sets.

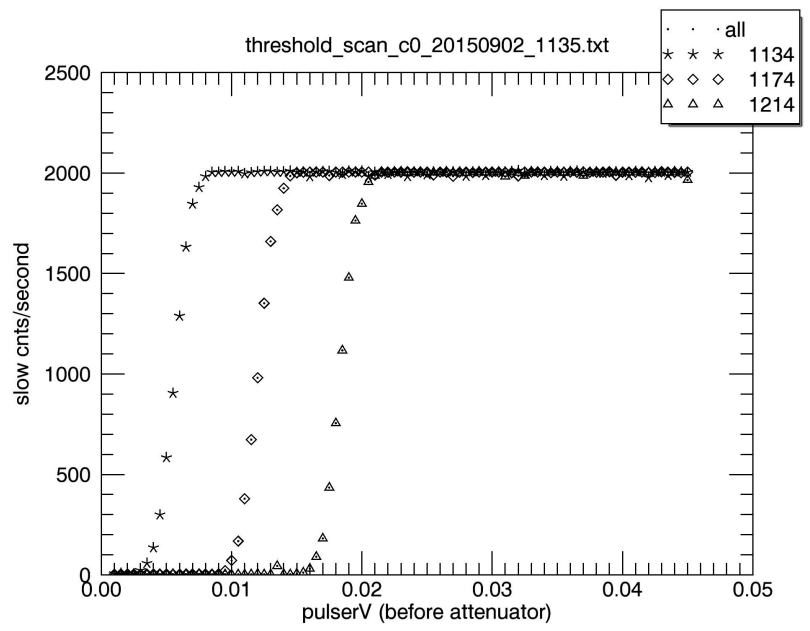

Figure 9. Pulser Scan: For each of three thresholds, input pulse height is varied.

\section{REFERENCES}

[1] Prigozhin, G., Gendreau, K., Arzoumanian, Z., J.Doty, Foster, R., Remillard, R., Malonis, A., LaMarr, B., Vezie, M., Egan, M., Villasenor, J., Baumgartner, W., Scholze, F., Laubis, C., Krumrey, M., and Huber, A., "Nicer instrument detector subsystem: Description and performance," Proc SPIE, 9905 (2016).

[2] Gendreau, K. et al., "The neutron star interior composition explorer (NICER): design and development," Proc SPIE, 9905 (2016).

[3] http://amptek.com/products/fast-sdd-silicon-drift-detector/.

[4] Gendreau, K., Arzoumanian, Z., Kenyon, S., and Spartana, N., "Minituarized high-speed modulated X-ray source," (US Patent 9,117,622 B2, 2015).

[5] Beckhoff, B., Gottwald, A., Klein, R., Krumrey, M., M?ller, R., Richter, M., Scholze, F., Thornagel, R., and Ulm, G., "A quarter-century of metrology using synchrotron radiation by PTB in Berlin," Physica Status Solidi, B 246, 1415-1434 (2009). 